\title{
Spatial variation of dynamic nuclear spin polarization probed by the non-local Hanle effect
}

J. Shiogai, M. Ciorga, M. Utz, D. Schuh, M. Kohda, D. Bougeard, T. Nojima, D. Weiss, and J. Nitta

Citation: Appl. Phys. Lett. 112, 132403 (2018); doi: 10.1063/1.5020314

View online: https://doi.org/10.1063/1.5020314

View Table of Contents: http://aip.scitation.org/toc/apl/112/13

Published by the American Institute of Physics

\section{Articles you may be interested in}

Effect of lithium doping on the optical properties of monolayer $\mathrm{MoS}_{2}$

Applied Physics Letters 112, 121902 (2018); 10.1063/1.5021629

A flexible optically re-writable color liquid crystal display

Applied Physics Letters 112, 131902 (2018); 10.1063/1.5021619

Atomically engineered epitaxial anatase $\mathrm{TiO}_{2}$ metal-semiconductor field-effect transistors

Applied Physics Letters 112, 133506 (2018); 10.1063/1.5024418

Gate-tunable quantum dot in a high quality single layer $\mathrm{MoS}_{2}$ van der Waals heterostructure Applied Physics Letters 112, 123101 (2018); 10.1063/1.5021113

Air-stable memory array of bistable rectifying diodes based on ferroelectric-semiconductor polymer blends Applied Physics Letters 112, 123302 (2018); 10.1063/1.5022864

Evolution of the spin hall magnetoresistance in $\mathrm{Cr}_{2} \mathrm{O}_{3} / \mathrm{Pt}$ bilayers close to the Néel temperature Applied Physics Letters 112, 132401 (2018); 10.1063/1.5019934

\section{Scilight} Sharp, quick summaries illuminating the latest physics research

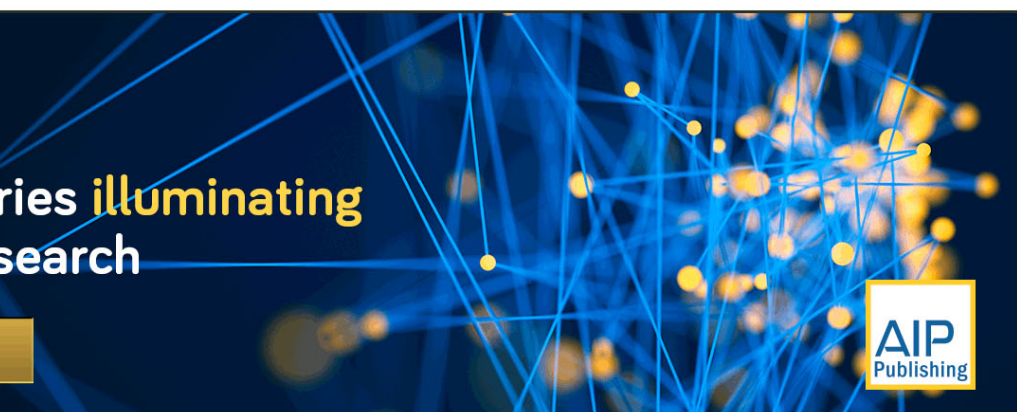




\title{
Spatial variation of dynamic nuclear spin polarization probed by the non-local Hanle effect
}

\author{
J. Shiogai, ${ }^{1, a)}$ M. Ciorga, ${ }^{2}$ M. Utz, ${ }^{2}$ D. Schuh,,${ }^{2}$ M. Kohda, ${ }^{1}$ D. Bougeard, ${ }^{2}$ T. Nojima, ${ }^{3}$ \\ D. Weiss, ${ }^{2}$ and J. Nitta ${ }^{1}$ \\ ${ }^{1}$ Department of Materials Science, Tohoku University, 980-8579 Sendai, Miyagi, Japan \\ ${ }^{2}$ Institute of Experimental and Applied Physics, University of Regensburg, D-93040 Regensburg, Germany \\ ${ }^{3}$ Institute of Materials Research, Tohoku University, 980-8577 Sendai, Miyagi, Japan
}

(Received 21 December 2017; accepted 10 March 2018; published online 27 March 2018)

\begin{abstract}
The spatial distribution of dynamic nuclear spin polarization (DNP) has been investigated in a lateral all-semiconductor spin-injection device based on a (Ga,Mn)As $/ n^{+}-\mathrm{GaAs}$ spin-Esaki diode. The DNP induced by the hyperfine interaction has been probed via satellite peaks in non-local Hanle-type spin precession signals, indicating the recovery of electron spin polarization. A quantitative analysis using the self-consistent calculation reproduces the magnetic field position of the satellite peaks as a function of spin injection bias and injector-detector separation. The distance dependence of the Hanle curves reveals that the spin-lattice relaxation rather than the hyperfine interaction is the dominant mechanism of nuclear spin relaxation in the non-local region. Published by AIP Publishing. https://doi.org/10.1063/1.5020314
\end{abstract}

In recent years, a comprehensive understanding of electrical spin injection from ferromagnets into lateral semiconductor structures and electrical non-local spin detection has been established, ${ }^{1,2}$ well described by the standard spin driftdiffusion model. ${ }^{3,4}$ Specifically, III-V semiconductor materials are good candidates for spintronic devices since the lack of inversion symmetry allows electrical tuning of spin precession through spin-orbit interactions. ${ }^{5-7}$ Spin-injection devices with a III-V semiconductor channel are inevitably affected by dynamic nuclear spin polarization (DNP) and associated electron spin relaxation due to hyperfine interaction because of non-zero nuclear spins. ${ }^{8-15}$ The hyperfine interaction is considered very useful for manipulating nuclear spins by electrical and optical means at relatively small external magnetic fields. ${ }^{16}$ It also allows us to detect nuclear spin polarization optically $^{8,9}$ or electrically ${ }^{11,12}$ through nuclear magnetic resonance (NMR) measurements. On the other hand, the nuclear magnetic field strongly modulates electron spin states, potentially hampering spintronic device applications. Actually, it has been reported that the DNP in a semiconductor channel strongly modulates the Hanle type spin precession. ${ }^{11,12,15,17}$ Therefore, the coupling strength between nuclear and electron spin systems, characterized by a leakage factor $f$, and its spatial variation in the pure spin current region are of great importance not only for in-depth understanding of DNP but also for actual applications of spin devices. A quantitative evaluation of the local variation of DNP along the channel has not been, however, clearly addressed so far in a resistive (electrical) detection method.

In this paper, we study the spatial variation of nuclear spin polarization in a lateral spin injection device using $(\mathrm{Ga}, \mathrm{Mn}) \mathrm{As} / n^{+}$-GaAs spin-Esaki diodes as spin injectors and spin detectors. ${ }^{2,18-20}$ The high spin injection/detection efficiencies and a long spin diffusion length allow us to map the

\footnotetext{
${ }^{\text {a) }}$ Author to whom correspondence should be addressed: junichi.shiogai@ imr.tohoku.ac.jp
}

distance dependence of the nuclear spin polarization in steps of $5 \mu \mathrm{m}$ up to $20 \mu \mathrm{m}$, with a large signal-to-noise ratio. The leakage factor $f$ was evaluated by performing self-consistent calculations based on the spin drift-diffusion equation, taking the nuclear magnetic field into account. ${ }^{12}$ When nuclear and external magnetic fields cancel, the Hanle curves exhibit satellite peaks, which are a direct measure of the nuclear magnetic field. ${ }^{11}$ The measurements and self-consistent analysis revealed that the nuclear spin polarization, induced by a pure spin current, is spatially inhomogeneous in the non-local region.

A schematic of the investigated device is depicted in Fig. 1(a). An electrical spin injection and detection device was fabricated from an epitaxial wafer consisting of a semiinsulating (001) GaAs substrate, a 0.3- $\mu \mathrm{m}$-thick GaAs buffer layer, a $0.5-\mu \mathrm{m}$-thick (Al,Ga)As/GaAs superlattice, a $0.8-\mu \mathrm{m}$ thick $n$-GaAs channel layer, 0.2- $\mu$ m-thick $n^{+}$-GaAs, a 15-nmthick $n^{+} \rightarrow n^{++}$-GaAs transition layer, 8.0-nm-thick $n^{++}$-GaAs, a 2.2-nm-thick low-temperature grown $(\mathrm{Al}, \mathrm{Ga})$ As diffusion barrier, and 50-nm-thick (Ga,Mn)As. The corresponding doping concentrations of each GaAs layer were $n=2 \times 10^{16} \mathrm{~cm}^{-3}$, $n^{+}=5 \times 10^{16} \mathrm{~cm}^{-3}$, and $n^{++}=6 \times 10^{18} \mathrm{~cm}^{-3}$, respectively. First, standard photolithography and wet chemical etching were used to define a 50- $\mu$ m-wide mesa oriented along the $y / /[110]$ direction. Then, ferromagnetic contacts along $x / /[1-10]$ were defined by electron beam lithography, Au/Ti evaporation, and the lift-off technique. The width of all the ferromagnetic contacts was $0.5 \mu \mathrm{m}$, and each center-to-center distance between neighboring contacts was $5 \mu \mathrm{m}$. Next, large contact pads were deposited at both ends of the mesa using photolithography and $\mathrm{Au} / \mathrm{Ti}$ evaporation. Finally, (Ga,Mn)As and the high doping layers down to $n$-GaAs were removed by reactive ion etching to leave a 0.8 - $\mu$ m-thick low doped $n$-GaAs transport channel. For performing non-local measurements, contact 2 was used as a spin injector, whereas other contacts 3, 4, 5, and 6 were employed as spin detectors at distances of $L=5$, 10,15 , and $20 \mu \mathrm{m}$ from the injector, respectively. The spin 
(a)
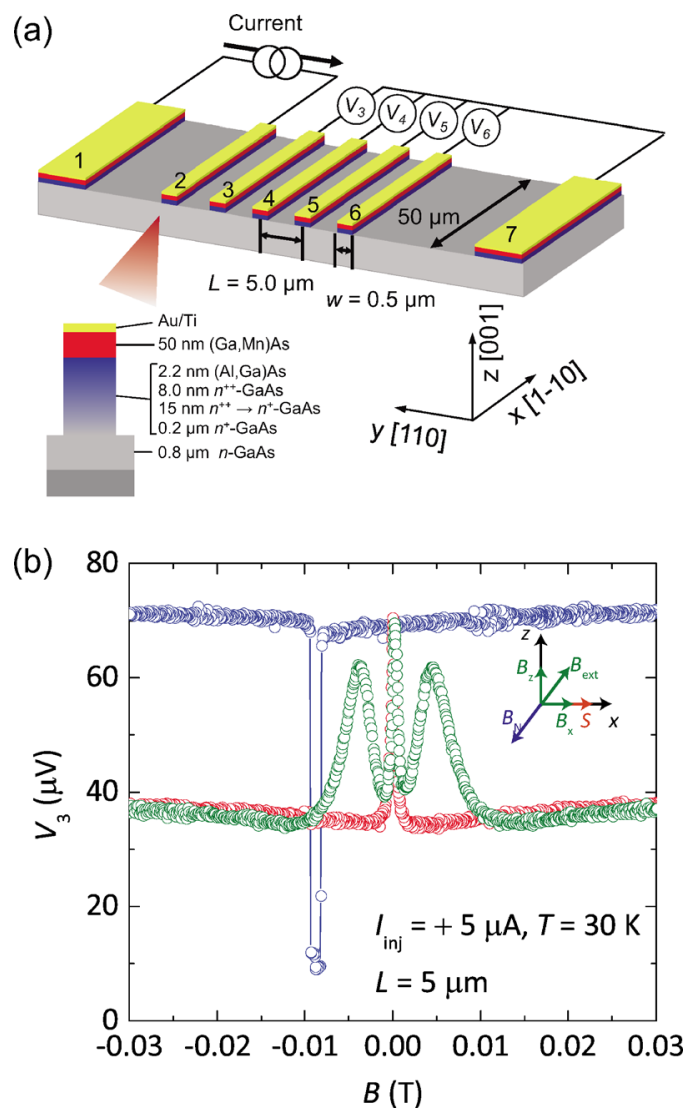

FIG. 1. (a) A schematic diagram of the spin-injection device. The transport channel is oriented along the [110] crystallographic direction, and ferromagnetic contacts are oriented along the [1-10] direction. The width of all small ferromagnetic contacts is $0.5 \mu \mathrm{m}$, and the center-to-center distance between neighboring contacts is $5 \mu \mathrm{m}$. The non-local voltages $V_{3}, V_{4}, V_{5}$, and $V_{6}$ were measured at $L=5,10,15$, and $20 \mu \mathrm{m}$, respectively. The cross-section of the used wafer is also shown. (b) The non-local voltage measured at the spin detector with $L=5 \mu \mathrm{m}$ and for $I_{\mathrm{inj}}=5 \mu \mathrm{A}$ and $T=30 \mathrm{~K}$ as a function of the in-plane magnetic field $B_{x}$ (the non-local spin-valve effect, blue circles) and the out-of-plane magnetic field $B_{z}$ (the Hanle effect). The Hanle curve was obtained without an additional in-plane component $B_{x}$ (red circles) and with $B_{\mathrm{x}}=+0.3 \mathrm{mT}$ (green circles). Inset: the configuration of the external magnetic field $\boldsymbol{B}_{\boldsymbol{e x}}$ and electron spin polarization $\boldsymbol{S}$ and nuclear magnetic field $\boldsymbol{B}_{\mathrm{N}}$ in the $x-z$ plane.

injection current $I_{\text {inj }}$ was driven between contacts 2 and 1. The non-local voltages $V_{3}, V_{4}, V_{5}$, and $V_{6}$ were measured with respect to the reference contact 7 .

Spin accumulation created underneath the injector diffuses into the non-local region, resulting in a pure spin current. Due to spin-charge coupling, a spin dependent electrochemical potential can be measured electrically. The nonlocal voltage measured at the detector with spin accumulation $\Delta \mu=\left(\mu_{\uparrow}-\mu_{\downarrow}\right) / 2$, with $\mu_{\sigma}$ being the electro-chemical potential for the spin channel $\sigma$, can be expressed as ${ }^{4,21,22}$

$$
V^{\mathrm{s}}=e \Delta \mu P_{d e t}= \pm \frac{e n P_{d e t}}{D\left(E_{F}\right)} P_{s}(L) .
$$

Here, $P_{\text {det }}$ is the spin detection efficiency, $n$ and $D\left(E_{F}\right)$ are the carrier concentration in the channel and the density of states at the Fermi level for a single spin channel, respectively, and $P_{\mathrm{s}}$ is the spin polarization in the channel, defined as $\left(n_{\uparrow}-n_{\downarrow}\right) /\left(n_{\uparrow}+n_{\downarrow}\right)$, with $n_{\sigma}$ being the spin density for the spin channel $\sigma$ and $e$ being the elementary charge. From nonlocal spin-valve measurements, $P_{\mathrm{det}} \sim 0.44$ was extracted.
Figure 1(b) shows typical results of non-local measurements at detector 3 with $L=5 \mu \mathrm{m}$ and the spin injection current $I_{\text {inj }}=+7 \mu \mathrm{A}$. In our definition, a positive value of $I_{\text {inj }}$ corresponds to spin extraction and a forward bias across the Esaki diode. A non-local spin-valve signal [blue circles in Fig. 1(b)] was clearly resolved upon sweeping an in-plane magnetic field $B_{\mathrm{x}}$ parallel to the long axis of the ferromagnetic contacts. Sweeping $B_{\mathrm{x}}$ from the positive saturation field through $B=0$ sequentially switches the magnetizations of the injector and the detector and results in the characteristic spin-valve pattern displayed by $V_{3}$. The upper and lower voltage values correspond to parallel and anti-parallel magnetization configurations, respectively. The voltage difference between these two magnetic configurations, denoted as $\Delta V_{\mathrm{NL}}$, is equal to $2 V^{\mathrm{s}}$. From the dependence of the non-local spin-valve signal $\Delta V_{\mathrm{NL}}$ on $L$ (not shown), $\lambda_{\text {sf }}$ was determined to be $7.6 \mu \mathrm{m}$. This value is comparable to that in our previous report. ${ }^{14}$ The regular Hanle curve [red circles in Fig. 1(b)] was obtained by sweeping the perpendicular magnetic field $B_{\mathrm{z}}$. The following procedure was used to minimize the effect of a nuclear field on the Hanle curves. First, $B_{\mathrm{x}}=+1 \mathrm{~T}$ was applied to magnetize the ferromagnetic injector and detectors in the plane, and then, $B_{\mathrm{x}}$ was reduced to zero. After waiting for $5 \mathrm{~min}, B_{\mathrm{Z}}$ was swept from zero to $+1 \mathrm{~T}$ at a rate of $5 \mathrm{mT} / \mathrm{min}$. Waiting time was needed in order to rule out time-dependent DNP effects. The typical value of nuclear spin relaxation time in our device is $2 \mathrm{~min}$ as demonstrated in our previous report with a similar carrier concentration in the channel. ${ }^{14}$ Actually, we confirmed that the non-local voltages do not change any more after $5 \mathrm{~min}$ of waiting time at zero field. The same procedure was used to sweep $B_{\mathrm{z}}$ from zero to $-1 \mathrm{~T}$ in order to complete a full Hanle curve measurement.

When the non-equilibrium accumulation of electron spins is generated by means of electrical spin injection, the nuclear spins also become dynamically polarized, as has been confirmed for our samples by NMR measurements. ${ }^{14}$ As a result of the hyperfine interaction between electron and nuclear spins, a nuclear magnetic field develops in the presence of an external magnetic field. The nuclear magnetic field $\boldsymbol{B}_{\mathrm{N}}$, to which the electron spins are exposed, at the steady state condition, for small spin polarization, neglecting the Knight shift and dipoledipole interactions between nuclear spins, is given by ${ }^{23}$

$$
\boldsymbol{B}_{\mathrm{N}}=f b_{N} \frac{\boldsymbol{S} \cdot \boldsymbol{B}_{e x}}{B_{e x}^{2}} \boldsymbol{B}_{e x},
$$

where $\boldsymbol{S}$ and $\boldsymbol{B}_{\text {ex }}$ are the locally varying electron spin polarization and the external magnetic field, respectively, $f \leq 1$ is a leakage factor, and $b_{\mathrm{N}}=-17 \mathrm{~T}$ in GaAs. ${ }^{23}$ The $f$ factor is given by the ratio of the nuclear spin relaxation rate due to the hyperfine interaction and the total nuclear spin relaxation rate as ${ }^{23}$

$$
f=T_{h f}^{-1} /\left(T_{h f}^{-1}+T_{1}^{-1}\right),
$$

where $T_{\mathrm{hf}}$ is the nuclear spin relaxation time due to the hyperfine interaction and $T_{1}$ represents other relaxation mechanisms. Here, we consider that $T_{1}$ is dominated by the nuclear spin-lattice relaxation. Note that $S=1 / 2$ when $P_{\mathrm{s}}=100 \%$ in our definition. 
The strength of the nuclear field can be estimated from Hanle measurements in the presence of an additional small in-plane field component $\boldsymbol{B}_{\boldsymbol{x}}$. In accordance with Eq. (2), the component of $\boldsymbol{B}_{\boldsymbol{e x}}=\boldsymbol{B}_{z}+\boldsymbol{B}_{\boldsymbol{x}}$ which is not orthogonal to the injected spin $\boldsymbol{S}$ leads to a non-zero $\boldsymbol{B}_{\mathrm{N}}$. Electron spins in the conduction band of the channel then experience the sum of the external magnetic field $\boldsymbol{B}_{\boldsymbol{e x}}$ and of the nuclear magnetic field $\boldsymbol{B}_{N}$. When the strength of $\boldsymbol{B}_{\text {ex }}$ is comparable and oriented opposite to the nuclear magnetic field $\left(\boldsymbol{B}_{\mathrm{ex}}=-\boldsymbol{B}_{\mathrm{N}}\right)$, the total magnetic field that electron spins see becomes zero so that the Hanle-type dephasing is strongly suppressed. This leads to the partial recovery of the electron spin polarization. As a result, satellite peaks are observed in Hanle curves, shown in Fig. 1(b) for $B_{\mathrm{x}}=+0.3 \mathrm{mT}$ (red circles). It is important to note that the non-local voltage, i.e., the electron spin polarization at the satellite peak, does not completely recover to the same voltage level as the main peak at $B_{\mathrm{z}}=0$, see Fig. 2(c). This implies that the magnetic field seen by the electron spins is the sum of the spatially homogeneous external magnetic field and the inhomogeneous nuclear magnetic fields.

The position of the satellite peak in the Hanle curves is a direct measure of an averaged value of the nuclear magnetic field $\boldsymbol{B}_{\mathrm{N}}$ that electron spins see while diffusing. ${ }^{11}$ According to Eq. (2), for a given $\boldsymbol{B}_{x}$, the nuclear field $\boldsymbol{B}_{\mathrm{N}}$ is proportional to $S$, i.e., $P_{\mathrm{S}}$, which depends on the injection current $I_{i n j}$ and temperature $T$. Actually, the peak position strongly depends on temperature, which reflects the temperature dependence of spin injection efficiency of a spin Esaki diode (not shown). ${ }^{2}$ Figure 2(a) shows the bias dependence of the electron spin polarization $P_{\mathrm{s}}$ at $L=5 \mu \mathrm{m}$, extracted from the spin valve signal $\Delta V_{\mathrm{NL}}=2 V^{\mathrm{s}}$ using Eq. (1). For positive $I_{\text {inj }}, P_{\mathrm{s}}$ monotonically increases up to $+7 \mu \mathrm{A}$ and then drops upon further increasing

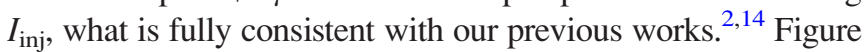
2(b) shows a color-coded plot of the non-local voltage as a function of $B_{\mathrm{z}}$ and $I_{\text {inj }}$ for fixed $B_{\mathrm{x}}=0.3 \mathrm{mT}$ and $T=30 \mathrm{~K}$, measured at $L=5 \mu \mathrm{m}$. Indeed, the position of the satellite

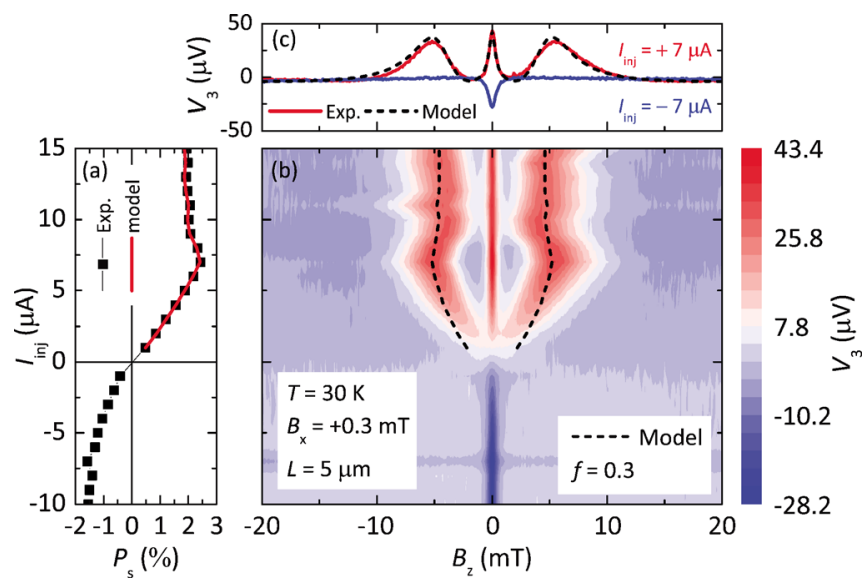

FIG. 2. (a) The electron spin polarization $P_{\mathrm{s}}$ at $L=5 \mu \mathrm{m}$ as a function of injection current $I_{\text {inj }}$ extracted from the non-local spin-valve signal (black squares). The self-consistent simulation reproduced $I_{\text {inj }}$ dependent $P_{\mathrm{s}}$ (red line). (b) Color mapping of non-local voltage $V_{3}$ as a function of $B_{\mathrm{z}}$ (horizontal axis) and $I_{\text {inj }}$ (vertical axis) measured at $T=30 \mathrm{~K}$ with $B_{\mathrm{x}}=+0.3 \mathrm{mT}$. The black dashed line indicates the satellite peak positions obtained by the simulation (see the text for details). (c) Two typical Hanle curves measured for $I_{\text {inj }}= \pm 7 \mu \mathrm{A}$ (red and blue lines). The simulated curve is plotted as a black dashed line. The background voltage has been subtracted from all the presented data. peaks follows this trend. The satellite peaks appear only when $I_{\text {inj }}>0$. To fulfill $\boldsymbol{B}_{\text {ex }}=-\boldsymbol{B}_{\mathrm{N}}$, the sign of $\boldsymbol{S} \cdot \boldsymbol{B}_{\mathrm{ex}}$ in the numerator of Eq. (2) needs to be positive, as $b_{\mathrm{N}}<0$ for GaAs. This means that for $I_{\text {inj }}>0, S$ is oriented along $\boldsymbol{B}_{\boldsymbol{x}}$ [see the inset of Fig. 1(b)]. Reversing the injection current changes the sign of $S$, and satellite peaks are no longer observed. This is shown in Fig. 2(c). Because the magnetization of the contacts $\boldsymbol{M}$ was saturated at $\boldsymbol{B}_{\mathrm{x}}=+1 \mathrm{~T}$ before the Hanle sweeps, the spins generated in (extracted from) the channel for $I>0$ are, as expected, parallel (antiparallel) to $\boldsymbol{M}^{24}$

The inhomogeneous distribution of the nuclear spin polarization in the channel was further confirmed by the dependence of the satellite peak position on the injector-detector separation $L$. Figure 3 shows the Hanle curves for $I_{\text {inj }}=+7 \mu \mathrm{A}$ measured at the detectors at distances of $L=5$, 10,15 , and $20 \mu \mathrm{m}$ from the injector (open circles). The amplitude of the main peak decreases with distance $L$ because of electron spin diffusion following $P_{\mathrm{s}}(L)=P_{\mathrm{s}}(0) \exp \left(-L / \lambda_{\mathrm{sf}}\right)$. In addition, the satellite peaks shift to smaller magnetic fields for larger $L$. We attribute such a distance dependence to the decay of the nuclear spin polarization with the increasing distance. Note that nuclear spin diffusion can be neglected because of the much smaller diffusion constant $D_{\mathrm{N}}=10^{-13} \mathrm{~cm}^{2} / \mathrm{s} \mathrm{com-}$ pared to electron spin diffusion. ${ }^{25}$ In order to quantitatively evaluate the spatial variation of the nuclear spin polarization and to determine the leakage factor $f$ in Eq. (2), we performed numerical calculations based on the one-dimensional spin drift diffusion model in the presence of a nuclear magnetic field

$$
\frac{\partial \boldsymbol{S}}{\partial t}=-v_{\mathrm{d}} \frac{\partial \boldsymbol{S}}{\partial y}+D \frac{\partial^{2} \boldsymbol{S}}{\partial y^{2}}-\frac{\boldsymbol{S}}{\tau_{\mathrm{sf}}}-\boldsymbol{\Omega}_{\mathrm{L}} \times \boldsymbol{S}
$$

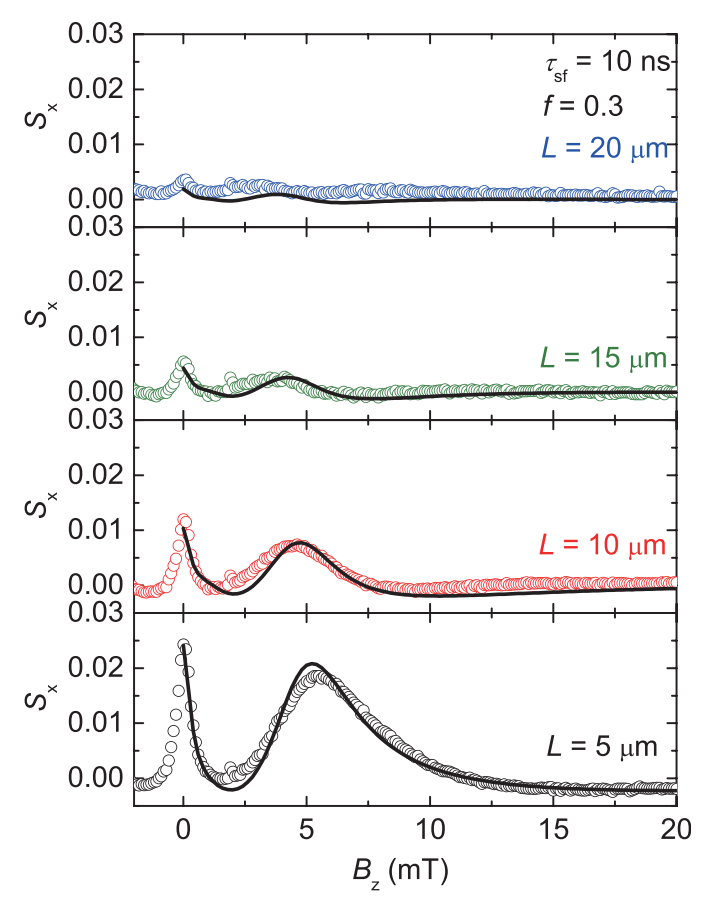

FIG. 3. Hanle curves, measured for $I_{\text {inj }}=+7 \mu \mathrm{A}$ and at $T=30 \mathrm{~K}$ (open symbols) at different detectors $L=5$ (black open circles), 10 (red), 15 (green), and $20 \mu \mathrm{m}$ (blue). For each $L$, the corresponding result of simulation, using $f=0.3$ and $\tau_{\mathrm{s}}=10 \mathrm{~ns}$, is plotted as a solid line. The vertical axis is re-scaled with the $x$-component of spin polarization $S_{\mathrm{x}}$, where $S=1 / 2$ for $P_{\mathrm{s}}=100 \%$ $\left[S_{\mathrm{x}}=V^{\mathrm{s}} D\left(E_{\mathrm{F}}\right) / 2 e n P_{\mathrm{det}}\right.$, with $V^{\mathrm{s}}$ being the experimentally measured non-local voltage, after subtraction of the background voltage]. 
Here, $v_{\mathrm{d}}$ is the drift velocity, $D$ is the spin diffusion constant, $\tau_{\mathrm{sf}}$ is the spin-relaxation time, and $\boldsymbol{\Omega}_{\mathrm{L}}$ represents the Larmor precession frequency about the effective magnetic field $\boldsymbol{B}_{\text {eff }}=\boldsymbol{B}_{e x}+\boldsymbol{B}_{N}$, i.e., the sum of the spatially dependent nuclear magnetic field $\boldsymbol{B}_{N}$ given by Eq. (2) and the external magnetic field $\boldsymbol{B}_{e x}$. The value of the spin polarization $\boldsymbol{S}$ varies along the channel. We determined $v_{\mathrm{d}}$ and $D$ from separate four-terminal resistance and Hall effect measurements. In the numerical calculations, we neglect the effect of stray fields from the (Ga.Mn)As ferromagnetic contacts, as the saturation magnetization of $(\mathrm{Ga}, \mathrm{Mn}) \mathrm{As}$ is relatively small in the investigated temperature range. ${ }^{26}$ As both Eqs. (2) and (4) are inter-dependent and non-linear equations, the solution needs to be obtained self-consistently. Here, the values of $f$ and $\tau_{\mathrm{sf}}$ are treated as fitting parameters. In Fig. 3, the best fits are plotted as solid lines, taking $f=0.3$ and $\tau_{\mathrm{sf}}=10 \mathrm{~ns}$ as common parameters for all $L$. The simulation reasonably reproduces the data. Such a value of $f$, slightly smaller than the one obtained in spin-LED structures with a non-doped quantum well, ${ }^{10}$ indicates that the nuclear spin relaxation time due to the hyperfine interaction is approximately 2 times longer than that due to the other mechanisms, such as nuclear spin-lattice relaxation. We ascribe such weakly coupling between nuclear and electron systems to the carrier concentration range being close to the metal-insulator transition in the present channel. For GaAs, the electron spins localized to a donor impurity are significantly influenced by nuclear spins in the insulating regime with lower carrier concentrations. ${ }^{9,27}$ On the other hand, the leakage factor of rather highly doped $n$-GaAs $\left(n=5 \times 10^{16} \mathrm{~cm}^{-3}\right)$ gives a smaller value on the order of $10^{-2}$ (Refs. 11 and 13). Comparison between the bias dependence of the satellite peak positions obtained from the simulation and the experiments is shown in Fig. 2, where the former is plotted as black dashed lines. Here, the simulation was performed by keeping $f=0.3$ and changing $P_{\mathrm{s}}$ at zero magnetic field to reproduce the non-local spin-valve measurements [red solid line in Fig. 2(a)]. In the regime of low spin accumulation for $I_{\text {inj }}<5 \mu \mathrm{A}$, the peak positions in experiments were slightly smaller than the simulated ones. This means that the actual value of $f$ is slightly dependent on $P_{\mathrm{s}}$ and smaller than that obtained by the simulation. It then follows that $T_{\mathrm{hf}}$ shows a monotonic dependence on $P_{\mathrm{s}}$ because of the relation $f=1 /\left(1+T_{\mathrm{hf}} / T_{1}\right)$ from Eq. (3) under the assumption that $T_{1}$ is constant. The distance dependence of the Hanle curves presented in Fig. 3 is consistent with this picture. The hyperfine-induced nuclear spin relaxation is driven by flip-flop spin scattering of electrons. Therefore, we consider that the non-equilibrium electron spin polarization would suppress the nuclear spin relaxation due to the hyperfine interaction although the microscopic origin is not fully understood yet. With increasing $L$, the deviation between the experiment and simulation becomes larger: the peaks shift towards smaller $B$. This also supports the idea that $P_{\mathrm{s}}$ and $f$ have a positive correlation.

In conclusion, we performed a systematic study on the spatial variation of the dynamically polarized nuclear spins by probing non-local Hanle signals at different distances $L$ from the point of injection. The experimental results suggest that the nuclear spin polarization has a strong spatial dependence in the non-local region of the spin injection channel within a length scale of a few $\mu \mathrm{m}$. The data can be explained by an inhomogeneous distribution of the nuclear field being induced by the spatially varying electron spin polarization. Moreover, from the self-consistent analysis based on the one-dimensional spin drift diffusion model in the presence of a nuclear magnetic field, we determined the leakage factor to be 0.3. This value indicates a weak hyperfine coupling between electron and nuclear spin systems in the non-local region.

This work was partly supported by the German Science Foundation (DFG) via SFB 689, the Japan-Germany Strategic International Cooperative Program (Joint Research Type) from JST and DFG (FOR 1483), Grants-in-Aid from JSPS $15 \mathrm{H} 05699$ and 24684019.

${ }^{1}$ X. Lou, C. Adelmann, S. A. Crooker, E. S. Garlid, J. Zhang, K. S. M. Reddy, S. D. Flexner, C. J. Palmstrøm, and P. A. Crowell, Nat. Phys. 3, 197 (2007).

${ }^{2}$ M. Ciorga, A. Einwanger, U. Wurstbauer, D. Schuh, W. Wegscheider, and D. Weiss, Phys. Rev. B 79, 165321 (2009).

${ }^{3}$ T. Valet and A. Fert, Phys. Rev. B 48, 7099 (1993).

${ }^{4}$ J. Fabian, A. Matos-Abiague, C. Ertler, P. Stano, and I. Zutić, Acta Phys. Slovaca 57, 565 (2007).

${ }^{5}$ G. Dresselhaus, Phys. Rev. 100, 580 (1955).

${ }^{6}$ Y. A. Bychkov and E. I. Rashba, J. Phys. C 17, 6039 (1984).

${ }^{7}$ J. Nitta, T. Akazaki, H. Takayanagi, and T. Enoki, Phys. Rev. Lett. 78, 1335 (1997).

${ }^{8}$ J. Strand, B. D. Schultz, A. F. Isakovic, C. J. Palmstrøm, and P. A. Crowell, Phys. Rev. Lett. 91, 036602 (2003).

${ }^{9}$ H. Sanada, S. Matsuzaka, K. Morita, C. Y. Hu, Y. Ohno, and H. Ohno, Phys. Rev. Lett. 94, 097601 (2005).

${ }^{10}$ J. Strand, X. Lou, C. Adelmann, B. D. Schultz, A. F. Isakovic, C. J. Palmstrøm, and P. A. Crowell, Phys. Rev. B 72, 155308 (2005).

${ }^{11}$ G. Salis, A. Fuhrer, and S. F. Alvarado, Phys. Rev. B 80, 115332 (2009).

${ }^{12}$ M. K. Chan, Q. O. Hu, J. Zhang, T. Kondo, C. J. Palmstrøm, and P. A. Crowell, Phys. Rev. B 80, 161206(R) (2009).

${ }^{13}$ C. Awo-Affouda, O. M. J. van't Erve, G. Kioseoglou, A. T. Hanbicki, M. Holub, C. H. Li, and B. T. Jonker, Appl. Phys. Lett. 94, 102511 (2009).

${ }^{14}$ J. Shiogai, M. Ciorga, M. Utz, D. Schuh, T. Arakawa, M. Kohda, K. Kobayashi, T. Ono, W. Wegscheider, D. Weiss, and J. Nitta, Appl. Phys. Lett. 101, 212402 (2012).

${ }^{15}$ T. Akiho, J. Shan, H.-X. Liu, K.-I. Matsuda, M. Yamamoto, and T. Uemura, Phys. Rev. B 87, 235205 (2013).

${ }^{16}$ M. I. D’yakonov and V. I. Perel', Zh. Eksp. Teor. Fiz. 60, 1954 (1971).

${ }^{17}$ N. J. Harmon, T. A. Peterson, C. C. Geppert, S. J. Patel, C. J. Palmstrøm, P. A. Crowell, and M. E. Flatté, Phys. Rev. B 92, 140201(R) (2015).

${ }^{18}$ M. Kohda, Y. Ohno, K. Takamura, F. Matsukura, and H. Ohno, Jpn. J. Appl. Phys., Part 2 40, L1274 (2001).

${ }^{19}$ E. Johnston-Halperin, D. Lofgreen, R. K. Kawakami, D. K. Young, L. Coldren, A. C. Gossard, and D. D. Awschalom, Phys. Rev. B 65, 041306 (2002).

${ }^{20}$ P. Van Dorpe, Z. Liu, W. Van Roy, V. F. Motsnyi, M. Sawicki, G. Borghs, and J. De Boeck, Appl. Phys. Lett. 84, 3495 (2004).

${ }^{21}$ M. Johnson and R. H. Silsbee, Phys. Rev. Lett. 55, 1790 (1985).

${ }^{22}$ A. Fert and H. Jaffrès, Phys. Rev. B 64, 184420 (2001).

${ }^{23}$ D. Paget, G. Lampel, B. Sapoval, and V. I. Safarov, Phys. Rev. B 15, 5780 (1977).

${ }^{24}$ T. Kuczmik, M. Oltscher, A. Bayer, D. Schuh, D. Bougeard, M. Ciorga, and D. Weiss, Phys. Rev. B 95, 195315 (2017).

${ }^{25}$ D. Paget, Phys. Rev. B 25, 4444 (1982).

${ }^{26}$ H. Ohno, A. Shen, F. Matsukura, A. Oiwa, A. Endo, S. Katsumoto, and Y. Iye, Appl. Phys. Lett. 69, 363 (1996).

${ }^{27}$ R. I. Dzhioev, K. V. Kavokin, V. L. Korenev, M. V. Lazarev, B. Y. Meltser, M. N. Stepanova, B. P. Zakharchenya, D. Gammon, and D. S. Katzer, Phys. Rev. B 66, 245204 (2002). 